Fortsetzung von der vorderen Klappe:

Im Herbst I96o wird erscheinen:

Erster Band:

Integration und Differentiation

der Funktionen einer Veränderlichen.

Anwendungen. Numerische Methoden.

Algebraische Gleichungen.

Unendliche Reihen

Dritte, verbesserte Auflage

Mit 169 Textabbildungen. X, 440 Seiten

Gr.-8. 1960

S 270.-, DM 45.-, sfr. 46.Io, \$10.70

Ganzleinen

S 288.—, DM 48.—, sfr. 49.10, \$1I.45

Aus den

Besprechungen zur zweiten Auflage:

„Der erste Band des in weiten Kreisen bekannten Werkes ist in der zweiten Auflage wieder erschienen. Alle Teile des ersten Bandes unterzog der Verfasser einer gründlichen Uberarbeitung; dabei wurden einige Abschnitte mit dem Ziel neu gefaßt oder ergänzt, eine ,wirklich lesbare Darstellung zu liefern, die aber doch jenes $\mathrm{MaB}$ von Strenge besitzt, das der Mathematiker nun eben einmal mit guten Gründen für unerläßlich hält‘. Man darf sagen, daß dieses Vorhaben gut gelang." Forschung auf dem Gebiet des Ingenieurwesens

\title{
Lieferbar ist:
}

Zweiter Band:

Integration und Differentiation der Funktionen von mehreren Veränderlichen. Lineare Algebra. Tensorfelder. Differentialgeometrie

$\mathrm{Zweite}$, neu bearbeitete Auflage

Mit 136 Textabbildungen

VIII, 401 Seiten. Gr.-80 1958

S 270.—, DM 45.-, sfr. 46.10, \$10.70

Ganzleinen

S 288. 一, DM 48.—, sfr. 49.10, \$ II.45

Band IV:

Reihenentwicklungen. Integralgleichungen. Laplace-Transformation.

Randwertprobleme. Potentialtheorie

Wird im Laufe des Jahres 196r erscheinen. 


\title{
Vorlesungen \\ über höhere Mathematik
}

\author{
Von
}

\section{Adalbert Duschek}

Vollständig in vier Bänden

Jeder Band ist einzeln käuflich

Erster Band

Integration und Differentiation

der Funktionen einer Veränderlichen.

Anwendungen. Numerische Methoden.

Algebraische Gleichungen.

Unendliche Reihen

Dritte, verbesserte Auflage

Mit 169 Textabbildungen. X, 440 Seiten Gr.-80. 1960

S 290.-, DM 46.—. sfr. 49.50, \$ Ir.50

Ganzleinen

$\mathrm{S}_{312 .-,} \mathrm{DM} 49.50$, sfr. $53.20, \$ 12.40$

Zweiter Band

Integration und Differentiation

der Funktionen von mehreren

Veränderlichen. Lineare Algebra.

Tensorfelder. Differentialgeometrie

Zweite, neu bearbeitete Auflage

Mit 136 Textabbildungen. VIII, 4 OI Seiten

Gr.-80. 1958

S 290.—, DM 46.—, sfr. 49.5o, \$ 11.50

Ganzleinen

S 312.-, DM 49.50, sfr. 53.20, \$1 2.40 


\section{Vorlesungen \\ über höhere Mathematik}

Von

\section{Adalbert Duschek}

Vollständig in vier Bänden

Jeder Band ist einzeln käuflich

Dritter Band

Gewöhnliche und partielle

Differentialgleichungen.

Variationsrechnung.

Funktionen einer komplexen

Veränderlichen

$\mathrm{Z}$ weite, mit Berichtigungen versehene Auflage

Mit 107 Textabbildungen. XII, 5I 2 Seiten Gr.-80. 1960

S 290.—, DM 46.—, sfr. 49.50, \$11.50

Ganzleinen

S 312.—, DM 49.50, sfr. 53.20, \$ I 2.40

\section{Vierter Band}

Integralgleichungen.

Laplacetransformation.

Randwertprobleme bei

gewöhnlichen Differentialgleichungen.

Grundzüge und Randwertaufgaben

der Potentialtheorie

Mit 49 Textabbildungen. VI, 335 Seiten Gr.-80. 196I

S 290.—, DM 46.—, sfr. 49.50, $\$ 11.50$

Ganzleinen

S 312.—, DM 49.50, sfr. 53.20, \$ 12.40 


\title{
Vorlesungen \\ über höhere Mathematik \\ Von
}

\section{Dr. phil. Adalbert Duschek}

weiland o. Professor der Mathematik an der Technischen Hodschule Wien

$$
\text { Dritter Band }
$$

Gewöhnliche und partielle Differentialgleichungen. Variationsrechnung. Funktionen einer komplexen Veränderlichen

\author{
Mit 107 Textabbildungen
}

$Z_{\text {weite, }}$ mit Beridhtigungen versehene Auflage

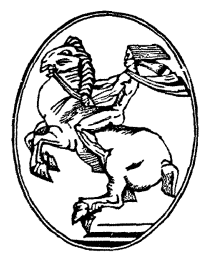

Wien

Springer $=$ Verlag

1960 
ISBN 978-3-7091-7688-7

ISBN 978-3-7091-7687-o (eBook)

DOI $10.1007 / 978-3-7091-7687-0$

Alle Rechte, insbesondere das der Übersetzung in fremde Sprachen, vorbehalten

Ohne ausdrückliche Genehmigung des Verlages ist es auch nicht gestattet, dieses Buch oder Teile daraus auf photomechanischem Wege (Photokopie, Mikrokopie) oder sonstwie $z u$ vervielfältigen

(C) I953 and 1960 by Springer-Verlag in Vienna Softcover reprint of the hardcover 2 nd edition 1960 


\title{
Vorwort zur ersten Auflage.
}

\author{
Alles ist von Wichtigkeit, \\ alles ist nicht gar so wichtig. \\ Nur die rechte Sichtigkeit \\ und $D u$ wandelst richtig.
}

Christian Morgenstern.

Ein Buch wie dieses ist in doppelter Hinsicht ein Kompromiß. Erstens ist es ein Kompromiß zwischen dem $\mathrm{Ma} ß$ an Strenge, das der Mathematiker liebt, und dem, das man dem Physiker und Techniker billigerweise zumuten darf; wie in den beiden ersten Bänden habe ich mich bemüht, vor allem die grundlegenden Begriffe und Aussagen möglichst klar und scharf herauszuarbeiten, während späterhin die Beweise mancher Sätze entweder gar nicht oder in einer Form gebracht werden, die keinen Anspruch auf volle Strenge erheben kann. $\mathrm{Daß}$ ich bei manchen Formulierungen die Voraussetzungen enger gefaßt habe, als es in einer für Mathematiker gedachten Darstellung zweckmäßig und üblich ist, bedarf wohl keiner weiteren Begründung.

Zweitens ist das Buch ein Kompromiß hinsichtlich der Auswahl des Stoffes. $\mathrm{Da}$ die drei Hauptthemen des Buches, Differentialgleichungen, Variationsrechnung und Funktionentheorie, zum unentbehrlichen Rüstzeug des Physikers und wissenschaftlich arbeitenden Technikers gehören, ist unbestritten. Strittig mag nur der Umfang sein, der einerseits durch den Plan des ganzen Werkes, anderseits aber zweifellos auch durch die subjektive Meinung des Verfassers bedingt ist. Jeden zufriedenzustellen mag wohl so gut wie ausgeschlossen sein. Ein abschließendes Urteil darüber, ob es mir gelungen ist, eine zweckmäßige Auswahl zu treffen, wird allerdings erst nach dem Erscheinen des vierten und letzten Bandes möglich sein.

Die meisten Werke, deren Ziele so weit gesteckt sind wie die des vorliegenden sind das Ergebnis der Zusammenarbeit mehrerer Verfasser. Das Resultat muß dann zwangsläufig ziemlich inhomogen werden, einem schillernden Mosaik vergleichbar, mit Überschneidungen da und Lücken dort. Macht sich ein einzelner an eine solche Aufgabe, so wird das Ergebnis zwar weniger schillern, aber dafür homogener sein. Beides hat wohl seine Vor- und Nachteile. Jedenfalls ist im zweiten Fall der Verfasser gezwungen, über Dinge zu schreiben, die seinem eigenen Arbeitsgebiet mehr oder weniger fern liegen, wo ihm daher die souveräne Beherrschung der Materie fehlt. Aber gerade bei einem Buch, das ein wirkliches Lehr-Buch sein will und sich nicht an den Spezialisten wendet, kann es im günstigsten Fall wohl sein, daß das nur ein Nachteil für den Verfasser, aber nicht für den Leser ist.

Aus dem Gesagten folgt mit Notwendigkeit, was aber doch ausdrücklich festgestellt sei : $\mathrm{Daß}$ ein Buch wie dieses dem Verfasser keine Extratouren persönlicher Vorlieben erlaubt und daher durchaus im Rahmen der vorhandenen und bewährten Literatur zu bleiben hat. Was die Differentialgleichungen betrifft, so muß ich in diesem Zusammenhang ganz besonders auf KAMkES „Differentialgleichungen der reellen Funktionen" verweisen. Ich glaube, daß heute eine Darstellung, 
die ernst genommen sein will, an KAMkEs Arbeit einfach nicht vorbeigehen kann, auch dann nicht, wenn sie sich ein wesentlich anderes Ziel gesetzt hat. Dazu kommt, daß ich - bedingt durch den Plan des ganzen Werkes - zwangläufig zur selben Beschränkung des Stoffes im Großen komme, die sich KAMKE freiwillig gibt: Zur Beschränkung auf das Reelle und auf die Diskussion von Anfangswertaufgaben; Randwertaufgaben werden nur gelegentlich gestreift und an einzelnen Beispielen, am häufigsten natürlich in der Variationsrechnung, behandelt. Aber KAMKE hat sein Buch für den Mathematiker geschrieben; dem Physiker bringt es zu viel - an minuziöser Strenge - und zugleich zu wenig: Es ist wohl fast selbstverständlich, daß ich beispielsweise den Begriff des allgemeinen Integrals nicht völlig unter den Tisch fallen lassen konnte und bei den partiellen Differentialgleichungen erster Ordnung die Theorie des vollständigen Integrals sogar ziemlich ausführlich bringen mußte.

In dem Abschnitt über Variationsrechnung habe ich mich grundsätzlich mit der Diskussion der notwendigen Bedingungen begnügt, obwohl vom Hilbertschen Unabhängigkeitssatz nur mehr ein verhältnismäßig kleiner Schritt zu den hinreichenden Bedingungen von WEIERSTRASS geführt hätte. Ziemlich ausführlich und bis zu einem gewissen Abschluß sind die Extremalenfelder und die Hamilton-Jakobische Theorie behandelt. Ein kleiner Exkurs über allgemeine Koordinaten und den Riemannschen Raum, im wesentlichen aus der Tensorrechnung von A. Hochrainer und mir übernommen, hat sich hier halbwegs zwanglos einfügen lassen.

Über den letzten Abschnitt, die Funktionentheorie, ist wenig zu sagen. Hier gibt es eine Fülle ausgezeichneter Monographien und daher ausgefahrene Geleise, die eine neue Darstellung wohl fast nur mehr im Rahmen eines umfassenderen Werkes rechtfertigen. Immerhin werden sich in Auswahl und Anordnung sowie in Einzelheiten doch einige neue, allerdings nicht sehr bedeutungsvolle Gesichtspunkte feststellen lassen, die im einzelnen aufzuzählen sich hier wohl erübrigt. Die Darstellung gipfelt in einer vielleicht etwas knapp gehaltenen, aber doch, wie ich hoffe, halbwegs umfassenden Darlegung der elliptischen Funktionen und Integrale.

Zum Schluß noch einige Hinweise für den Leser. Das Buch setzt selbstverständlich eine gute Kenntnis der Differential- und Integralrechnung voraus, ist aber trotz vieler Hinweise auf die beiden ersten Bände, die lediglich der Bequemlichkeit derjenigen dienen, die diese besitzen, von ihnen durchaus unabhängig und für sich lesbar. Jeder der drei großen Abschnitte, in die das Buch zerfällt, beginnt in einer gewissen Breite und Ausführlichkeit, aber in dem Maß, als die Darstellung in die Tiefe vordringt, werden die Anforderungen an die selbständige Mitarbeit des Lesers größer. Man möge aber nicht den Mut verlieren, wenn man da und dort eine Entwicklung nicht auf den ersten Anhieb völlig versteht, sondern zunächst einmal weiterlesen und sich den betreffenden Abschnitt wieder und dafür gründlicher vornehmen, wenn zum erstenmal darauf zurückverwiesen wird. Manches wird der Anfänger bei einem ersten Studium zunächst einmal ganz überschlagen können, wie z. B. die Beweise der Sätze in den Ziffern 5 und 6 von $\S 3$, die Ziffern 7 und 8 von $\S$ I2 usw. Mit zwei Ausnahmen finden sich am Schluß jedes Paragraphen einige Übungsaufgaben mit mehr oder weniger ausführlichen Lösungen am Schluß des Bandes; ich empfehle aber sehr, die Lösung nur zur Kontrolle des eigenen Resultates oder zumindest erst dann anzusehen, wenn man wirklich nicht mehr weiter kann. Einige etwas schwierigere Aufgaben sind wieder durch einen Stern gekennzeichnet.

Dem Buch ist ein kleines Literaturverzeichnis (LV) beigefügt, das vor allem die wichtigsten im deutschen Sprachgebiete verbreiteten Werke enthält; das Verzeichnis macht natürlich keinerlei Anspruch auf Yollständigkeit und soll nur einige An- 
regungen zur Weiterbildung und Vertiefung des Wissens über das hier Gebrachte hinaus vermitteln.

Für aufopfernde Hilfe bei der sauren Arbeit des Korrekturenlesens, für viele wertvolle Hinweise und Verbesserungsvorschläge habe ich den Herren Dr. W. Eberl, Professor Dr. H. Hornich, Dr. L. Peczar, Privatdozent Dr. L. Schmetterer sowie meiner Frau, die wieder das ganze Manuskript geschrieben hat, herzlich zu danken. Mein Dank gebührt auch der Druckerei für ihre ausgezeichnete Arbeit und dem Verlag für sein großzügiges Eingehen auf meine Wünsche.

Wien, im Frühjahr I953.

A. Duschek.

\section{Vorwort zur zweiten Auflage.}

In der zweiten Auflage wurden Korrekturen und Verbesserungen vorgenommen, die noch vom Verfasser selbst vorbereitet worden waren.

Die Hinweise auf die Bände I und II beziehen sich in dieser Auflage von S. I28 an bereits auf die neu bearbeiteten zweiten Auflagen und damit auch auf alle etwaigen weiteren Auflagen von Band I und II. Bis zur S. I28 wurden die auf die zweiten Auflagen abgeänderten Hinweise nur als Berichtigungen gebracht.

Wien, im Herbst Ig6o.

F. Duschek. 


\section{Inhaltsverzeichnis.}

\section{Gewöhnliche Differentialgleichungen erster Ordnung. Seite}

$\S$ I. Einleitende Bemerkungen über Differentialgleichungen im allgemeinen .........

I. Der Begriff der Differentialgleichung. - 2. Differentialgleichungen und Funktionalgleichungen. - 3. Naturgesetze und Differentialgleichungen. - 4. Die Struktur der Lösungen gewöhnlicher Differentialgleichungen. - 5. Das Auftreten willkürlicher Funktionen in den Lösungen partieller Differentialgleichungen.

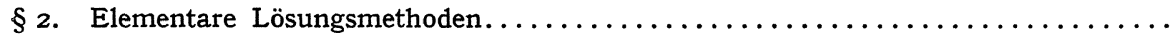

I. Das Richtungsfeld einer gewöhnlichen Differentialgleichung. - 2. Die exakten Differentialgleichungen. - 3. Die Differentialgleichung mit getrennten Variablen. - 4. Differentialgleichungen, die in $x$ und $y$ homogen sind. - 5. Die lineare Differentialgleichung. - 6. Die Riccatische Differentialgleichung. - 7. Die implizite Differentialgleichung. - 8. Singuläre Integrale. - 9. Die Differentialgleichungen von D'Alembert und Clairaut. - io. Singuläre Punkte einer Differentialgleichung.

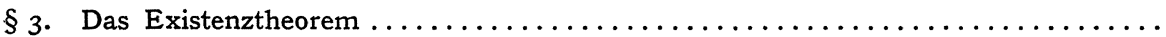

I. Vorbemerkungen. - 2. Das Verfahren der sukzessiven Approximationen. 3. Das Existenztheorem. Ergänzungen. - 4. Das allgemeine Integral. - 5. Verhalten der Lösungen bei kleinen Änderungen der Differentialgleichung. - 6. Die Abhängigkeit der Lösungen von den Anfangsbedingungen.

§ 4. Graphische und numerische Lösungsmethoden ....................

I. Graphische Integration. - 2. Das Verfahren der sukzessiven Approximationen. - 3. Integration durch Reihen. - 4. Das Verfahren von Runge-Kutta.

\section{Gewöhnliche Differentialgleichungen höherer Ordnung.}

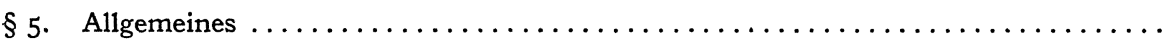

I. Differentialgleichungen höherer Ordnung und Systeme von Differentialgleichungen erster Ordnung. - 2. Geometrische Deutung und graphische Lösungsverfahren. - 3. Existenz und Eindeutigkeit der Lösungen. - 4. Näherungsverfahren. - 5. Elementar integrierbare Fälle. - 6. Die Differentialgleichung der Kettenlinie.

$\S 6$. Die allgemeine lineare Differentialgleichung $\ldots \ldots \ldots \ldots \ldots \ldots \ldots \ldots \ldots$

I. Allgemeines. Existenz der Lösungen. - 2. Lineare Abhängigkeit von Funktionen einer Veränderlichen. Die Wronskische Determinante. - 3. Die Struktur der Lösungen der homogenen Gleichung. - 4. Integration der homogenen Gleichung. Reduktionsverfahren von D'AlEmberT. - 5. Das allgemeine Integral der inhomogenen Gleichung. Die Variation der Konstanten.

§ 7. Die lineare Differentialgleichung mit konstanten Koeffizienten ............

I. Die homogene Gleichung. - 2. Die inhomogene Gleichung. - 3. Die Eulersche Gleichung.

§. Die Schwingungsgleichung

I. Mechanische Schwingungen. - 2. Elektrische Schwingungen. - 3. Periodische Störungsfunktionen. - 4. Die Resonanzkurve. - 5. Die komplexe Rechnung in der Elektrotechnik.

§ 9. Systeme linearer Differentialgleichungen $\ldots \ldots \ldots \ldots \ldots \ldots \ldots \ldots \ldots \ldots \ldots$

I. Allgemeines. Existenz der Lösungen. - 2. Die Struktur der Lösungen des homogenen Systems. - 3. Inhomogene Systeme. Variation der Konstanten. 4. Lineare Systeme erster Ordnung mit konstanten Koeffizienten. - 5. Die Operatorenrechnung von HEaviside und die linearen Systeme höherer Ordnung. - 6. Fortsetzung. Inhomogene Gleichungen. 
$\S$ ro. Vorbemerkungen $\ldots \ldots \ldots \ldots \ldots \ldots \ldots \ldots \ldots \ldots \ldots \ldots \ldots \ldots \ldots \ldots \ldots \ldots \ldots \ldots$ I 4

I. Funktionaldeterminanten und Abhängigkeit von Funktionen. - 2. Hüllflächen ein- und zweiparametriger Flächenscharen. - 3. Flächenelement und Streifen.

$\S$ II. Lineare partielle Differentialgleichungen erster Ordnung $\ldots \ldots \ldots \ldots \ldots \ldots \ldots$ I22

r. Definitionen. - 2. Die homogene Gleichung mit zwei unabhängigen Veränderlichen. - 3. Fortsetzung. Konstruktion einer Lösung durch eine gegebene Kurve. Allgemeines Integral. - 4. Die allgemeine homogene Gleichung. - 5. Die inhomogene Gleichung. - 6. Überbestimmte Systeme partieller Differentialgleichungen.

$\S$ I2. Die allgemeine partielle Differentialgleichung erster Ordnung

I. Geometrische Deutung und Charakteristiken. - 2. Eine andere Deutung der charakteristischen Gleichungen und der Begriff des Vorintegrals. - 3. Konstruktion einer Integralfläche durch eine gegebene Kurve. - 4. Über die Integration der charakteristischen Gleichungen. - 5. Das vollständige Integral. - 6. Einige Sonderfälle. - 7. Die allgemeine Gleichung mit $n$ unabhängigen Veränderlichen. 8. Ermittlung eines vollständigen Integrals aus $m$ Vorintegralen.

§ 13. Partielle Differentialgleichungen zweiter Ordnung ................... I6I

I. Streifen zweiter Ordnung und charakteristische Streifen. - 2. Die lineare Gleichung zweiter Ordnung. - 3. Die homogene lineare Differentialgleichung mit konstanten Koeffizienten. - 4. Die Methode der partikulären Lösungen von D. Bernoulli. - 5. Die Kaskadenmethode von Laplace bei hyperbolischen Differentialgleichungen. - 6. Adjungierte Differentialausdrücke und die Greensche Formel.

$\S$ I4. Die hyperbolische Differentialgleichung $\ldots \ldots \ldots \ldots \ldots \ldots \ldots \ldots \ldots \ldots \ldots \ldots \ldots \ldots$ I $\mathrm{I}$

I. Die beiden Arten von Anfangsbedingungen. - 2. Existenzsätze. - 3. Die Riemannsche Integrationsmethode. - 4. Die Differentialgleichung der schwingenden Saite und die Telegraphengleichung.

\section{Variationsrechnung.}

$\S$ I5. Die Eulersche Differentialgleichung $\ldots \ldots \ldots \ldots \ldots \ldots \ldots \ldots \ldots \ldots \ldots \ldots \ldots \ldots \ldots \ldots \ldots$

I. Problemstellung, Beispiele. - 2. Ein Hilfssatz. - 3. Die Eulersche Differentialgleichung im einfachsten Fall. - 4. Einige ergänzende Bemerkungen. 5. Sonderfälle und Beispiele. - 6. Das inverse Problem der Variationsrechnung.

$\S$ I6. Allgemeinere Variationsprobleme, Ergänzungen ................... I99

I. Das Auftreten höherer Ableitungen in der Grundfunktion. - 2. Mehrere unbekannte Funktionen. - 3. Variationsprobleme in Parameterdarstellung. 4. Fortsetzung. Die Normalform der Eulerschen Gleichungen. - 5. Diskontinuierliche Lösungen. - 6. Der allgemeine Fall variabler Endpunkte und die Transversalitätsbedingung. - 7. Extremalenfelder. - 8. Der Hilbertsche Unabhängigkeitssatz. - 9. Die Transversalitätsbedingung bei $n$ abhängigen Veränderlichen.

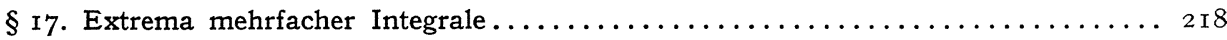

r. Doppelintegrale. - 2. Das Dirichletsche Problem. - 3. Dreifache Integrale. - 4. Doppelintegrale in Parameterdarstellung. - 5. Minimalflächen.

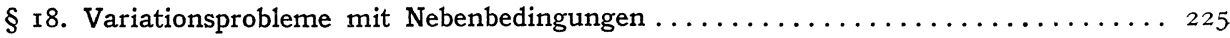

I. Isoperimetrische Probleme. - 2. Endliche oder holonome Bedingungsgleichungen. - 3. Anholonome Bedingungsgleichungen.

$\S$ I9. Allgemeine Koordinaten und allgemeine Räume $\ldots \ldots \ldots \ldots \ldots \ldots \ldots \ldots \ldots$

I. Vorbemerkungen. - 2. Allgemeine krummlinige Koordinaten im euklidischen $R_{3}$. - 3. Kontravariante und kovariante Koordinaten eines Vektors. 4. Tensoren höherer Stufe in allgemeinen Koordinaten. - 5. Vektoren und Tensoren in allgemeinen Räumen. - 6. Der Riemannsche Raum. - 7. Der Eulersche Vektor eines homogenen Variationsproblems. - 8. Die geodätischen Linien im Riemannschen Raum. - 9. Über die Riemannsche Geometrie. - Io. Die Invarianz der Eulerschen Gleichungen. - I I. Transformationen von $\Delta z$ auf allgemeine Koordinaten. 
I. Variationsprinzipe in der Physik. - 2. Das ebene Problem. - 3. Fortsetzung. Das vollständige Integral der Hamilton-Jacobischen Differentialgleichung. 4. Der allgemeine Fall. Die Methode von CARATHEODORY. - 5. Einführung kanonischer Variabler und die Hamilton-Jacobische Differentialgleichung. - 6. Das allgemeine Integral der Eulerschen Gleichungen und das vollständige Integral der Hamilton- Jacobischen Gleichung. - 7. Kanonische Transformationen. - 8. Das Hamiltonsche Prinzip in der Dynamik. - 9. Die Planetenbewegung und die Keplerschen Gesetze.

\section{Die regulären Funktionen einer komplexen Variablen.}

$\S 2$. Komplexe Zahlen und Punktmengen in der Ebene .............. 275

I. Komplexe Zahlen. - 2. Die Gaußsche Zahlenebene. - 3. Die stereographische Projektion, die Riemannsche Zahlenkugel und der Punkt $z=\infty$. 4. Punktmengen in der Gaußschen Zahlenebene. - 5. Der Borelsche Überdeckungssatz. - 6. Punkt- und Zahlenfolgen, unendliche Reihen. - 7. Die Abelsche Reihentransformation. - 8. Einige Sätze über Potenzreihen.

$\S 22$. Der Begriff der regulären Funktion $\ldots \ldots \ldots \ldots \ldots \ldots \ldots \ldots \ldots \ldots \ldots \ldots \ldots$

I. Komplexe Funktionen einer komplexen Veränderlichen. - 2. Grenzwert einer Funktion. - 3. Stetigkeit und gleichmäßige Stetigkeit. - 4. Die Ableitung einer komplexen Funktion. - 5. Die Differentialgleichungen von CAUCHY-RiemanN und LAPLACE. Der erste Fundamentalsatz der Funktionentheorie. - 6. Zusammenhang mit der Strömungslehre. - 7. Die inverse Funktion. - 8. Exponentialfunktion und Logarithmus.

§ 23. Die konforme Abbildung

I. Reguläre Funktionen und konforme Abbildung. - 2. Die bilineare Funktion. 3. Die Fixpunkte der bilinearen Transformationen. - 4. Die Funktion $w=z^{2}$ und der Begriff der Riemannschen Fläche. - 5. Logarithmus und allgemeine Potenz.

$\S 24$. Die Integration der komplexen Funktionen $\ldots \ldots \ldots \ldots \ldots \ldots \ldots \ldots \ldots \ldots \ldots$

I. Der Begriff des bestimmten Integrals und die Integrierbarkeit der stetigen Funktionen. - 2. Einige einfache Integralsätze. - 3. Der zweite Fundamentalsatz der Funktionentheorie und das unbestimmte Integral. - 4. Mehrfach zusammenhängende Gebiete. Der Residuensatz. - 5. Die Cauchysche Intcgralformel. 6. Folgerungen aus der Integralformel. - 7. Isolierte singuläre Punkte. 8. Integrale mit einem Parameter. - 9. Zur Berechnung bestimmter Integrale.

$\S 25$. Reihen und Reihenentwicklungen ...................... $33^{8}$

I. Konvergenz und gleichmäßige Konvergenz. - 2. Gleichmäßig konvergente Reihen regulärer Funktionen. - 3. Der dritte Fundamentalsatz der Funktionentheorie. - 4. Nullstellen, a-Stellen und Kreuzungspunkte. - 5. Der Abelsche Stetigkeitssatz. - 6. Der Weierstraßsche Doppelreihensatz. - 7. Die Laurentsche Entwicklung. - 8. Pole und wesentlich singuläre Stellen einer Funktion. 9. Folgerungen. - Io. Einteilung der Funktionen.

$\S 26$. Der Begriff der analytischen Fortsetzung und das vollständige analytische Gebilde

I. Der Identitätssatz für reguläre Funktionen. - 2. Der Begriff der analytischen Fortsetzung und der Monodromiesatz. - 3. Die analytische Fortsetzung durch Potenzreihen. - 4. Mehrdeutige Funktionen und Riemannsche Flächen. -. 5. Das Prinzip der Stetigkeit. - 6. Das Schwarzsche Spiegelungsprinzip.

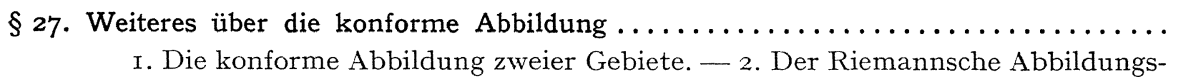
satz. - 3. Konforme Abbildung eines Polygons auf die Halbebene. - 4. Die Abbildung eines Dreiecks. - 5. Die Abbildung eines Rechtecks. - 6. Ebene Felder.

$\S 28$. Das Poissonsche Integral und die erste Randwertaufgabe der Potentialtheorie . 380

I. Das Poissonsche Integral. - 2. Die Randwerte einer harmonischen Funktion. - 3. Das Maximum-Minimum-Prinzip und die Reihenentwicklung harmonischer Funktionen. 
§ 29. Ganze Funktionen

I. Ganze rationale Funktionen und der Fundamentalsatz der Algebra. 2. Ganze transzendente Funktionen. - 3. Unendliche Produkte. - 4. Der Weierstraßsche Produktsatz.

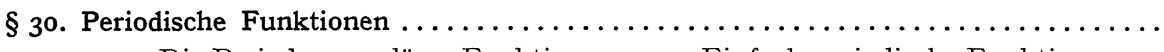

I. Die Perioden regulärer Funktionen. - 2. Einfach periodische Funktionen. 3. Fortsetzung: Die rationalen Funktionen von $\exp \gamma z$. - 4. Fortsetzung: Das Verhalten an den Enden des Periodenstreifens. - 5. Doppelt periodische Funktionen.

$\S 3$ I. Die meromorphen Funktionen

I. Teilbruchreihen. - 2. Der Satz von Mittag-Leffrler. - 3. Funktionen mit lauter einfachen Polen. - 4. Die Partialbruchentwicklung von $\cot \pi z$. - 5. Herleitung des Weierstraßschen Produktsatzes aus dem Satz von Mitrag-Leffrer.

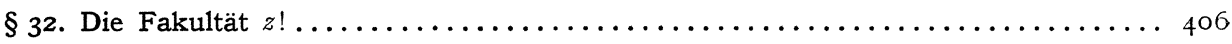

I. Die Darstellungen von Euler und Gauss. - 2. Die Darstellung von $\mathrm{I} / z$ ! durch ein unendliches Produkt. - 3. Folgerungen. - 4. Identität der Ausdrücke von Euler und Gauss. - 5. Darstellung von $z$ ! durch ein Kurvenintegral. - 6. Das Eulersche Integral erster Art.

$\S$ 33. Die elliptischen Funktionen

I. Allgemeine Eigenschaften. - 2. Die Weierstraßsche $\wp$-Funktion. - 3. Die Differentialgleichung der $k$-Funktion. - 4. Die Funktionen $\zeta(z)$ und $\sigma(z)$. 5. Die allgemeine Darstellung der elliptischen Funktionen durch die $\sigma$-Funktion. - 6. Folgerungen. - 7. Das elliptische Gebilde.

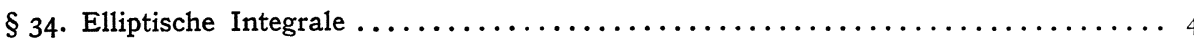

I. Die drei Grundtypen elliptischer Integrale. - 2. Bilineare Transformationen. - 3. Die Weierstraßschen Normalformen. - 4. Bestimmte Integrale. - 5. Die Normalintegrale von Riemann und Legendre. - 6. Die Perioden der Weierstraßschen und Legendreschen Normalintegrale. - 7. Das mathematische Pendel. 8. Zur Überführung elliptischer Integrale in die Legendresche Normalform.

$\S 35$. Die $\vartheta$-Funktionen und die elliptischen Funktionen Jacobis............... 444

I. Überblick. Die Weierstraßschen $\sigma$-Funktionen. - 2. Die vier $\vartheta$-Funktionen. 3. Einige Folgerungen. - 4. Die elliptischen Funktionen JACOBIS. - 5. Weitere Formeln für die elliptischen Funktionen JAcoBis. - 6. Lineare Transformationen der Perioden. - 7. Die Transformation der elliptischen Funktionen. - 8. Die Transformation der Legendreschen Normalintegrale. - 9. Die Landensche Transformation.

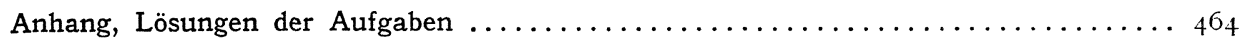

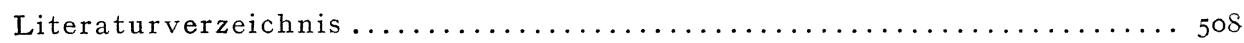

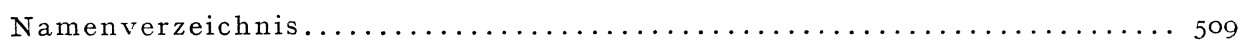

Sachverzeichnis ........................................... 509 


\section{Berichtigungen.}

S. 50, Zeile 2 von unten lies: Integralgleichung (Io), statt: Integralgleichung (7).

S. 67 , Zeile 2 von unten lies: I $+\sum_{\nu=1}^{\infty}$ statt: $\sum_{\nu=0}^{\infty}$.

S. 79, Zeile 4 vor Ziffer 5 lies: $\frac{(-\mathrm{I})^{\nu-1}}{2^{2} 4^{2} \ldots(2 v)^{2}}$ statt: $\frac{(-\mathrm{I})^{\nu-1} x^{2 \nu}}{2^{2} 4^{2} \ldots(2 v)^{2}}$.

S. 99, Zeile 4 nach (Io) lies: $\stackrel{0}{y}_{i}$ statt: $\stackrel{0}{y}_{j}$.

S. I07, zu Beginn der Zeile 4 von oben schalte ein: $(\gamma=0$ kann willkürlich gewählt werden).

S. II5, Zeile 3 nach (5) lies: „,Bereiche ..., wenn der ...“ statt: ,Figuren ..., wenn die ...".

S. II5, Zeile 2 nach (6) lies: „Bereiche“ statt: „Figuren“.

S. II6, Zeile 2 vor (II) lies: ,im p-dimensionalen Raum der $x_{1}, \ldots, x_{p}$ “ statt: ,im $n$-dimensionalen Raum der $x_{1}, \ldots, x_{n}$ “.

S. I24, Zeile 5 von oben lies: $(\alpha, \beta)$ statt $[\alpha, \beta]$.

S. I26, Zeile 6 vor Ziffer 4 lies: $Q: P$ statt $P: Q$.

S. I62, Zeile 3 nach (5) lies: ,... also die ersten fünf Funktionen (3) mit der Bedingung (4) gegeben sind, die drei restlichen aus (I) und (5) berechnen können, ..."

statt: ,... also die ersten fünf Funktionen (3) gegeben sind, die drei restlichen aus (I), (4) und (5) berechnen können, ....".

S. 280, Zeile I von unten lies: eine stückweise glatte Kurve“ statt: ,eine stetige und stückweise glatte Kurve".

S. 3I5, Zeile 5 von unten lies: ,, bilineare Transformation“ statt: ,lineare Transformation".

S. 328, Zeile 6 nach (30) lies: $|f(z)|<M$ statt: $f(z)<M$.

S. 37I, Zeile I nach (7): ,wodurch $A$ bestimmt ist" ist zu streichen.

S. 395, Zeile 2 von § 30 lies: „Pole“ statt: ,singuläre Stellen“.

S. 396, Zeile II von unten lies: „Pole“ statt: „Singularitäten“.

S. 398, Zeile 8 von unten lies: „Pole“ statt: „,singuläre Stellen“. 


\section{Berichtigungen von Hinweisen.}

S. 34, Zeile 8 von unten lies: II, $\S 8,6$ statt: II, $\S$ I3, 6 .

S. 38, Zeile 7 von unten lies: II, $\S 8,2$ statt: II, § I3, 2.

S. 56, Zeile Io von oben lies: II, 55 statt: II, $\S$ II.

S. 56, Zeile I5 von oben lies: I, § 34, 8 statt: II, $§ 2$.

S. 56, Zeile I7 von oben lies: I, $\S 37$, I statt: II, $\S 5$, I.

S. 58, Zeile 5 von unten lies: I, $\S 27,3$ statt: I, $\S 35,3$.

S. 6o, Zeile I von unten lies: Band II, I. Auflage, S. 234 statt: Band II, S. 234.

S. 63, Zeile I2 von unten lies: II, $\S 8,4$ statt: II, $\S$ I3, 4 .

S. 73, Zeile I5 von oben lies: I, $\S 29,5$ statt: I, § 37, 5 .

S. 78, Zeile I nach (2I) lies: I, § I5, 4 statt: I, § I8, 4 .

S. 9I, Zeile I9 von oben lies: I, § 39 statt: II, $\S 6$.

S. 9I, Zeile 2 I von oben lies: I, § 39, 4 statt: II, §6, 4 .

S. 95, Zeile I4 von oben lies: I, § I7, 4 statt: I, § 23, 4.

S. 97, Zeile I7 von unten lies: I, § I6, I2 statt: I, § 22, II.

S. I04, Zeile 4 von oben lies: II, $\S$ I8 statt: II, $\S 28$.

S. I05, Zeile I von oben lies: II, $\S$ I8 statt: II, $\S 28$.

S. I05, Zeile 5 von oben lies: II, § I6, 2 statt: II, $\S 26,2$.

S. I05, Zeile I3 von unten lies: II, § I8, 3 statt: II, § 28, 3 .

S. IIo, Zeile 6 von oben lies: II, $\S$ I5, 5 statt: II, $\S 25,5$.

S. II2, Zeile 4 von oben lies: II, §II, 2 statt: II, § I6, 3 .

S. II3, Zeile 4 von oben lies: II, $\S$ I6, I statt: II, $\S 26$, I.

S. II4, Zeile II von unten lies: II, § 7 statt: II, $\S$ I2.

S. II6, Zeile 3 von oben lies: II, § I7, 5 statt: II, §27, 4 .

S. II6, Zeile 6 von unten lies: II, $\S 8,6$ statt: II, $\S$ I3, 6 .

S. II9, Zeile 9 von oben lies: II, $\S 5,5$ statt: II, $\S$ II, 5 . 